\title{
Sentential and discourse topic effects on lexical ambiguity processing: An eye movement examination
}

\author{
KATHERINE S. BINDER \\ Mount Holyoke College, South Hadley, Massachusetts
}

\begin{abstract}
Models of lexical ambiguity resolution posit a role for context, but this construct has remained relatively undefined in the literature. The present study isolated two different forms of contextual constraint and examined how these sources of information might differentiate between a selective access and a reordered access model of ambiguity processing. Eye movements were monitored as participants read passages that contained either a balanced or a biased ambiguous word. The sentence containing the ambiguous word was held constant and instantiated either the subordinate meaning (Experiment 1) or the dominant meaning (Experiment 2) through the use of local context. These sentences were embedded in passages in which the topic was consistent, inconsistent, or neutral with respect to the meaning biased by the critical sentence. Experiment 1 provided evidence suggesting that the subordinate meaning of an ambiguous word was not selectively accessed even when sentence and discourse topic information biased that meaning. The data from Experiment 2 provided evidence that even the dominant meaning was not selectively accessed. These contextual sources of information were evaluated in terms of the roles they play in models of lexical ambiguity resolution.
\end{abstract}

Much research has been devoted to examining how contextual information influences the processing of lexically ambiguous words. Most of this research on lexical ambiguity, however, has focused on how ambiguous words are processed in sentences; little empirical work has been devoted to examining how discourse factors influence the process of lexical ambiguity resolution. As an individual reads a passage, many sources of information contribute to the intended message of the text. While a person is reading an individual sentence, the syntactic structure as well as the semantic content may influence the processing of an individual word. As sentences are strung together, however, a discourse-level representation emerges that may incorporate information not explicitly represented in the individual sentences of that passage, such as a reader's general world knowledge about a specific situation. Models of lexical ambiguity resolution differ in how they portray the role of context, but none of them deals with how different sources of context are used during the resolution process. That is, what are the constraints on how different sources of information combine

This research was part of a doctoral dissertation conducted at the University of South Carolina under the direction of Robin K. Morris and was supported by Grant BNS-9110115 from the National Science Foundation to Robin K. Morris. In addition, preparation of this manuscript was supported by an NIMH postdoctoral fellowship. I thank Robin K. Morris, A. René Schmauder, Keith Rayner, Susan Duffy, David Gorfein, and an anonymous reviewer for helpful comments and suggestions on earlier drafts. Correspondence should be addressed to K. S. Binder, Department of Psychology and Education, Mount Holyoke College, 50 College St., South Hadley, MA 01075 (e-mail: kbinder@ mtholyoke.edu). to influence word processing? The experiments reported here were designed to investigate how information within a sentence is combined with discourse-level topic information to influence the resolution of lexical ambiguity.

In his review of the literature, Simpson (1984) classified models of ambiguity processing into three general categories: selective, exhaustive, and ordered access models. According to the selective access account, prior biasing context works to activate only the contextually relevant meaning of an ambiguous word (Glucksberg, Kreuz, \& Rho, 1986; Oden \& Spira, 1983; Paul, Kellas, Martin, \& Clark, 1992; Perfetti \& Goodman, 1970; Schvaneveldt, Meyer, \& Becker, 1976; Simpson, 1981; Simpson \& Krueger, 1991; Swinney \& Hakes, 1976; Tabossi, 1988; Tabossi, Colombo, \& Job, 1987). For example, consider the following sentence: "There were several insects, roaches, and other bugs in the room." A selective access account suggests that the prior context-the reference to insects and roaches-activates only the contextually appropriate meaning when the word bugs is encountered during reading or hearing of the sentence. Thus, the prior context exerts a top-down influence by activating only the appropriate meaning of the ambiguous word.

According to exhaustive access accounts (Conrad, 1974; Kintsch \& Mross, 1985; Lucas, 1987; Onifer \& Swinney, 1981; Seidenberg, Tanenhaus, Leiman, \& Bienkowski, 1982; Swinney, 1979; Tanenhaus, Leiman, \& Seidenberg, 1979; Till, Mross, \& Kintsch, 1988), upon the appearance of a lexically ambiguous word, all its meanings are accessed regardless of preceding biasing context. This initial stage of multiple access is followed by the selection of the contex tually appropriate meaning. 
Support for this view has been gained primarily through the use of the cross-modal priming paradigm. In this task, participants are presented auditorily with a sentence that contains an ambiguous word. Immediately following the offset of the ambiguous word, a target word appears on a computer screen to which the participant must make some sort of response, usually a naming or lexical decision response. When the target is presented immediately after the ambiguous word, responses to either meaning of the ambiguous word are facilitated relative to those for a neutral control word, but after a short delay, facilitation is found only for the contextually appropriate meaning (Burgess, Tanenhaus, \& Seidenberg, 1989; Kintsch \& Mross, 1985; Onifer \& Swinney, 1981; Seidenberg et al., 1982; Swinney, 1979; Tanenhaus et al., 1979; Till et al., 1988).

Several other studies have provided evidence consistent with the notion that both context and meaning frequency play a role in the resolution of lexical ambiguity (e.g., Binder \& Morris, 1995; Dopkins, Morris, \& Rayner, 1992; Duffy, Morris, \& Rayner, 1988; Neill, 1989; Neill, Hilliard, \& Cooper, 1988). For example, according to the reordered access model (Duffy et al., 1988), all meanings of an ambiguous word are accessed, but the order in which they are accessed is influenced by meaning dominance as well as prior context. In a number of eye movement studies, researchers have obtained evidence consistent with the reordered access account (Binder \& Morris, 1995; Binder \& Rayner, 1998; Dopkins et al., 1992; Duffy et al., 1988; Kambe, Rayner, \& Duffy, 2001; Rayner, Binder, \& Duffy, 1999; Rayner \& Duffy, 1986; Rayner \& Frazier, 1989; Rayner, Pacht, \& Duffy, 1994; Sereno, 1995; Sereno, Pacht, \& Rayner, 1992). These researchers monitored readers' eye movements as they read sentences and measured fixation time on the ambiguous word or a control word that was matched in length and frequency. The findings from these studies can be summarized as follows. When neutral context precedes the ambiguous word, readers fixate balanced ambiguous words (words that have two equally likely meanings) longer than biased words (words that have one dominant interpretation) or an unambiguous control word. However, readers spend significantly more time in the disambiguating region following the target word in the case of biased words that are disambiguated toward the subordinate interpretation. When the disambiguating information precedes the ambiguous word, the pattern of results is quite different. Fixation times are longer for the biased ambiguous words when the preceding context biases toward the subordinate meaning than for either the balanced words or unambiguous control words. The reordered access model suggests that in the case of balanced ambiguous words, the resting activation of the two meanings is roughly equivalent. The context that precedes the ambiguous word works to boost the activation of the contextually relevant meaning so that it is accessed prior to the contextually inappropriate meaning. Lexical access time is not increased, because no subsequent selection process is required. The contextually appropriate meaning is integrated with the prior context in the same manner as it is for the control words. In the case of biased ambiguous words, however, the preceding biasing context boosts the activation of the less frequent meaning so that it is accessed at or near the same time as the dominant interpretation. The resulting inflated processing times on these words are due to a time-consuming selection process between the meanings. Rayner et al. (1994) referred to this finding as the subordinate bias effect.

The preceding discussion of models of ambiguity resolution indicates that context plays a role in the resolution process. Although numerous studies have demonstrated that sentence context may facilitate access of the contextually appropriate meaning of an ambiguous word (e.g., Dopkins et al., 1992; Duffy et al., 1988; Paul et al., 1992; Simpson \& Krueger, 1991; Tabossi, 1988; Tabossi et al., 1987; Tabossi \& Zardon, 1993), there is much less evidence regarding the ambiguity resolution process in more extended discourse. That is, as individual sentences are placed in a sequence to form a passage, additional contextual sources of information emerge. This discourselevel information comes from the meaning of the text in conjunction with the comprehender's knowledge of the situation referred to by the text. It is possible that discourselevel information activates higher order memory structures that reflect general world knowledge about specific situations and that therefore may enhance processing for words that are conceptually related to the higher order structures independently of any enhanced lexical processing attributable to the contextual information found within the sentence containing the target word.

It has been argued that global discourse topic information influences early stages of word processing for unambiguous target words (Schwanenflugel \& White, 1991; A. J. Sharkey \& N. E. Sharkey, 1992; N. E. Sharkey $\&$ Mitchell, 1985). These studies manipulated script- and schema-related information (N. E. Sharkey \& Mitchell, 1985), as well as topic information (Schwanenflugel \& White, 1991). N. E. Sharkey and Mitchell (1985) and A. J. Sharkey and N. E. Sharkey (1992) demonstrated that distant information from a script passage could facilitate lexical decisions for script-related words, regardless of filler sentences not directly referring to the script that were interspersed between the script-activating sentence and the script-related word. These researchers have suggested that there are several different representational forms of linguistic knowledge, but these factors work together via a general cognitive mechanism to influence early stages of processing (Schwanenflugel \& White, 1991; A. J. Sharkey \& N. E. Sharkey, 1992; N. E. Sharkey $\&$ Mitchell, 1985). Thus, information contained within a single sentence, as well as higher order representations such as script-based or topic-related information, directly facilitate access of lexical representations. In addition, Vu, Kellas, Metcalf, and Herman (2000) have argued that discourse-level representations, in the form of pronominal reference, constrain meaning activation of 
an ambiguous word in such a way that selective access occur.

These studies differ from eye movement studies, which have examined the influence of discourse-level information. Rayner et al. (1994) examined the processing of biased ambiguous words in passages in an attempt to test between a selective access and a reordered access account of ambiguity resolution. Rayner et al. (1994) monitored participants' eye movements as they read passages in which they manipulated whether the participants had previously encountered a biased ambiguous word instantiated in its subordinate sense. They measured gaze durations on the second encounter of the biased ambiguous word or an unambiguous control word. According to the selective access view, meaning repetition should make the subordinate meaning more available, thereby reducing processing difficulty on the homograph. However, in spite of word repetition, processing times were inflated on the ambiguous word relative to the control word, suggesting that both meanings were accessed, and a time-consuming selection process occurred. However, later processing measures were decreased when the meaning was repeated across the passage, suggesting that the discourse context influenced the integration of the meaning into the discourse representation.

Binder and Morris (1995) also investigated how a prior instance of an ambiguous word influenced later processing of that word. They monitored eye movements as participants read passages that contained two occurrences of a balanced ambiguous word. The meaning of the ambiguous word either remained the same or changed across the course of the passage from the first to the second encounter. In addition, the initial topic of the passage was either maintained or shifted between the two occurrences of the ambiguous word. The results demonstrated that meaning repetition facilitated lexical access regardless of discourse structure, whereas the discourse structure influenced text integration, but had no effect on initial processing.

These eye movement studies (Binder \& Morris, 1995; Rayner et al., 1994) failed to provide evidence that discourse-level contextual information is able to exert a top-down influence in such a way that access is limited to only the contextually appropriate meaning. Thus, there was no early influence of discourse context. These studies do suggest that discourse-level contextual variables influence word processing, but the influence is felt at later stages of word processing, such as meaning selection and/or meaning integration. One difference between the eye movement studies and the studies showing an early influence of discourse information (Schwanenflugel \& White, 1991; A. J. Sharkey \& N. E. Sharkey, 1992; N. E. Sharkey \& Mitchell, 1985; Vu et al., 2000) lies in the choice of discourse information. The former studies used ambiguity repetition within a passage, whereas the latter relied upon topic-, script-, or schema-related information. It is possible that the latter form of contextual information is stronger and is therefore able to produce a top- down influence on ambiguity processing in such a way that selective access occurs. This possibility was investigated in the present experiments.

Two experiments were conducted to assess the effects of (1) the inherent meaning bias of an ambiguous word, (2) the target sentence context, and (3) the discourse-level topic information within a passage on lexical ambiguity resolution. Thus, the primary objective of this study was to develop a more principled account of how different sources of contextual information influence lexical ambiguity resolution. In order to accomplish this, participants' eye movements were monitored so that the influence of these different sources could be examined as word processing unfolded over time. In each of the experiments, the local context of the sentence containing the ambiguous word was held constant. In Experiment 1, the target sentence context established a bias consistent with the subordinate meaning of the ambiguous word, whereas in Experiment 2 , the lexical information instantiated the dominant meaning of the ambiguous word. These critical sentences were then embedded in passages in which the topic information was consistent with the subordinate interpretation, consistent with the dominant interpretation, or neutral with respect to either meaning.

In order to examine how these different contextual variables influenced word processing, different regions of the text and different processing measures from the eye movement record were examined. The regions examined were the target word and the posttarget region, which extended from the end of the target word to the end of the critical sentence. First-fixation duration and gaze duration were the eye movement measures that were used to assess processing differences in each of the regions. Using multiple regions and multiple on-line processing measures allowed for an assessment of the temporal course of word processing. Although no single measure extracted from the eye movement record can be taken as an absolute, "pure" measure of lexical access or text integration, this methodology does provide an online record whereby changes in processing over time can be examined.

\section{EXPERIMENT 1}

In Experiment 1, the influence of different sources of information (meaning dominance, context of the critical sentence containing the ambiguous word, and discourselevel topic information) on word processing was examined. In this experiment, participants read passages that contained either a biased (one dominant meaning) or a balanced (both meanings equally likely) ambiguous word. The ambiguous words were placed in critical sentences that established a bias consistent with the subordinate meaning, and the biasing contextual information always preceded the target word. For example, the target sentence context of the passage in Table 1 consisted of contextually biasing items such as money, bags, and federal, which were intended to bias toward the subordinate 
Table 1

Example Passages from Experiment 1: Target Sentence Context Is Always Biased Toward Subordinate Meaning

Topic Consistent

Mugsy and Molly contemplated the fortune that would soon be theirs. Mugsy scratched his head and nervously shifted his feet. Molly gripped her purse tightly enough to whiten her knuckles. They stuffed the money in their bags and walked out of the federal mint/jail without being caught.

Topic Inconsistent

Mugsy and Molly were so hungry they could hardly stand it. Mugsy scratched his head and nervously shifted his feet. Molly gripped her purse tightly enough to whiten her knuckles. They stuffed the money in their bags and walked out of the federal mint/jail without being caught.

Topic Neutral

Mugsy and Molly contemplated the big step they were about to take. Mugsy scratched his head and nervously shifted his feet. Molly gripped her purse tightly enough to whiten her knuckles. They stuffed the money in their bags and walked out of the federal mint/jail without being caught.

Note-The ambiguous target word is italicized in the last sentence and is followed by its matched control word.

sense of the ambiguous word mint. The critical sentences containing the ambiguous words were embedded in passages in which the topic established in the first sentence of the passage was manipulated. The intent of the first sentence was to establish a topic that was consistent with the subordinate meaning (e.g., contemplating a fortune: topic consistent), consistent with the dominant meaning (e.g., being hungry: topic inconsistent), or neutral with respect to either meaning (e.g., contemplating a big step: topic neutral). It is important to note that the context of the target sentence did not shift the topic of the passage even when the meaning biased in that sentence was different from the meaning biased in the initial sentence of the passage (in the topic-inconsistent condition).

The topic-neutral condition was included in order to assess the effects from the local content of the sentence in the absence of discourse-level topic information. Therefore it was expected that this condition would replicate the subordinate bias effect found in the aforementioned eye movement studies using single sentences. The other topic conditions were included to assess how the pattern of data would be altered as a function of discourse information. If word processing is sensitive to topic information, then alterations in the subordinate bias effect could occur as a function of topic bias. This effect could manifest itself in at least two different ways. First, consistent topic information might reduce or eliminate the subordinate bias effect in the topic-consistent condition relative to the topic-neutral condition. That is, the combination of topic and target sentence context biasing toward the subordinate meaning could override the inherent processing difficulty associated with the subordinate meaning of biased ambiguous words. Conversely, the subordinate bias effect could be exaggerated in the topic-inconsistent condition because the topic information is inconsistent with the local context relative to the topic-neutral condition.
Here, two pieces of information, the meaning dominance of the word and topic information, point to the dominant interpretation, whereas the target sentence context is consistent with the subordinate interpretation. Thus, the topic-inconsistent condition is most likely to expose topic effects on initial processing since two sources of information, meaning dominance and topic information, are consistent with the dominant interpretation and are inconsistent with the subordinate interpretation. Finally, processing measures on the target word were examined since these measures are primarily thought to reflect initial stages of processing, and the posttarget region was examined in order to assess the impact of the different sources of information on later stages of word processing.

\section{Method}

Participants. Thirty-six University of South Carolina students received either class credit or payment for participation in the experiment. The sample reflected a general cross section of the university community, which consists of both undergraduate and graduate students. All participants had uncorrected vision and were native English speakers.

Apparatus. Eye movements were recorded by a Fourward Technologies Dual Purkinje Image Eyetracker 1000 that was interfaced with a PS/2 Model 80 IBM computer. The eyetracker has a resolution of $10^{\prime}$ of arc. Although viewing was binocular, only the right eye was monitored. The signal from the eye tracker was sampled every millisecond by the computer. The passages were presented on an IBM VGA color monitor, and the participants were seated $79 \mathrm{~cm}$ from the monitor with four characters of text subtending $1^{\circ}$ of visual angle. The brightness of the screen was adjusted for each participant, to ensure comfort.

Procedure. Participants were told that they would be reading a series of passages presented on a computer screen. They were told to read for comprehension and that from time to time they would be asked a question about the passage they had just read, which could be answered by responding "yes" or "no." They were asked comprehension questions on approximately $25 \%$ of the passages. After each participant understood the procedure and informed consent was obtained, a bite bar was prepared in order to minimize head movement. The eyetracking system was then calibrated to the participant. This procedure took approximately $5 \mathrm{~min}$ for each participant.

At the beginning of each trial, five boxes appeared on the screen and the participant was instructed to look at the left-most box. Once the experimenter had determined that the participant was fixating the box, the entire passage was presented on the screen to begin the trial. When the participant was finished reading the passage, he/she was instructed to push a button that ended that trial. The passage was then replaced by the five boxes. As soon as the participant was ready to read the next passage, he/she was told to look at the leftmost box, and the experimenter presented the next passage. This procedure was repeated for the 8 practice passages and the 72 experimental passages that each participant read.

Materials. Thirty-six critical words were selected from norms collected at the University of South Carolina as well as other norms (Duffy et al., 1988; Gorfein, Viviani, \& Leddo, 1982; Twilley, Dixon, Taylor, \& Clark, 1994). Eighteen biased (one highly dominant interpretation) and 18 balanced (both meanings equally likely) ambiguous words were chosen for the experiment. The dominant sense of the biased words had a probability range of .79-.99, with a mean of .89 . The dominant sense of the balanced ambiguous words had a probability range of .43-.62, with a mean of .52. Each ambiguous word was paired with an unambiguous word, which was matched in length and frequency according to the Francis and Kučera (1982) norms. ${ }^{1}$ 
The average word frequency count for the biased words was 59 per million (range: 1-242); the average length was 4.7 characters (range: $3-7)$. The average word frequency for their matched control words was 55 (range: 2-230); the average word length was 4.7 (range: 3-7). The average frequency of the balanced ambiguous words was 54 per million (range: 1-286); the average length was 5.1 (range: 4-7). The average frequency for their matched control words was 58 (range: 1-302); the average length was 5.1 (range: 4-7).

Each ambiguous word was embedded in a sentence that was biased toward the subordinate meaning of the ambiguous word. The biasing sentence context always preceded the target word. The target sentence was in turn placed in a passage as the final sentence of that passage. Control conditions were created by replacing the ambiguous target word with an unambiguous word of equal length and frequency, which also fit into the sentence context (see norming task, below). The topic of the discourse passage was established in the initial sentence of the passage, and this sentence established a topic that was consistent with the subordinate meaning of the ambiguous word (topic consistent), consistent with the dominant meaning (topic inconsistent), or neutral with respect to either meaning (topic neutral). This initial topic sentence was followed by two intervening filler sentences. These two filler sentences were the same across all versions of the passage, and they were neutral in the sense that they were not incongruous continuations of the initial sentence regardless of the topic condition.

Twelve within-subjects conditions were formed by crossing the three factors of meaning dominance (biased or balanced), topic (consistent, inconsistent, or neutral), and word type (ambiguous target or control word). These factors were counterbalanced using a Latin-square design, and the order of presentation was randomized for each participant. Each participant read a total of 72 experimental passages and saw every ambiguous word as well as its matched control word. However, the words were each presented in different passage frames. Thus, no target word, control word, or passage frame was ever repeated for a given participant.

To verify that the ambiguous words and control words fit equally well into the local sentence context, two norming tasks were conducted. For the first norming task, two versions of a booklet were created, with each version containing 72 sentences. Half of the items in each booklet contained the sentence with the ambiguous word, and half contained a sentence with the unambiguous control word. In a given booklet, the participant saw either the sentence with the ambiguous word or the sentence with the control word. The items were randomized within the booklet. The target word, whether it was the ambiguous word or the control word, was presented in boldface type. The participant's task was to indicate on a scale of 1 to 7 how well the word fit into the sentence context $(1=$ not at all; $7=$ very good fit). Thirty Mount Holyoke College students participated in this task. The average rating for the sentences containing the ambiguous word was 6.1 , and the average rating for the sentences containing the control word was 5.8. A $t$ test used to test this difference showed that the difference was not significant $(p>.05)$. Thus, it appears that the control words fit into the sentence context just as naturally as do the ambiguous target words.

In order to create a more stringent test of naturalness, a second norming task was conducted. Twenty-f ive Mount Holyoke College students participated in a rating task in which they saw the 72 pairs of sentences and were asked to rate the relative naturalness of the two sentences in each pair. They were to indicate whether the sentence with the ambiguous word seemed more natural, the sentence with the unambiguous control word seemed more natural, or whether they were equally natural. (The participants were not told that one sentence contained an ambiguous word. In addition, the order of the two sentences in a pair was randomized.) The three categories were coded as $1,-1$, and 0 , respectively, and the mean rating was used for a given sentence as the rating of the naturalness of that sentence. The mean value for this measure was 0.021 , which in- dicates that the sentences, on the average, were almost perfectly balanced in terms of the word's fitting into the context of the sentence. On the basis of the results of these two norming tasks, it is clear that both words fit equally well into the local sentence context.

To ensure that (1) the topic sentences established a bias toward the intended meaning of the ambiguous word, and (2) the topic-consistent and topic-inconsistent conditions were equally biasing toward their respective meanings, an additional norming task was conducted. In this task, 30 Mount Holyoke College students were presented with booklets that contained the topic sentences from each of the three conditions, although there were three different versions of the booklet, so that each participant saw one version for each of the items. The conditions were counterbalanced across booklets, and the items were randomized within the booklet. The booklets contained the topic sentence followed by the ambiguous word and two associates, one for each of the noun-noun meanings. Additionally, a 7-point rating scale was provided. The participants were told to (1) circle the associate corresponding to the meaning of the ambiguous word that was indicated by the sentence context and (2) rate on a scale of 1 to 7 how biasing the sentence context was toward the meaning that they had selected $(1=$ not very biasing; and $7=$ very biasing $)$. When the topic sentence biased the dominant meaning of the ambiguous word, its associate was chosen $97 \%$ of the time. The subordinate associate was chosen $98 \%$ of the time when the topic sentence biased subordinate meaning. Further, when the topic neutral sentences were presented, the associate related to the dominant meaning was circled $57 \%$ of the time, and the subordinate associate was chosen $43 \%$ of the time. Thus, according to this aspect of the norming task, the topic sentences were successful in that they biased the intended meaning of the ambiguous word, but the topic neutral sentences did not reliably bias one meaning over the other. The results of the rating task for the dominant biasing, subordinate biasing, and neutral topic sentences were $6.3,6.1$, and 1.6, respectively. There was no significant difference between the topic sentences for the dominant and subordinate meaning ( $t$ test, $p>.05$ ), but both of these conditions were rated as more biasing than the neutral condition (all $p \mathrm{~s}<.01$ ).

\section{Results}

Several aspects of processing were examined: firstfixation and gaze duration on the target word itself and the gaze duration on the region that extended from the end of the target word to the end of that sentence (posttarget region). First-fixation duration is the duration of the first fixation on the word regardless of how many times the reader fixated the word. Gaze duration is the sum of all consecutive fixations on a word before the reader's gaze leaves that word. This measure does not include any regressions to the target word from other regions of the text. If the target word was not fixated, the closest fixation within three character spaces to the left and one character space to the right of the target was counted as the fixation during which the target word was processed (Rayner \& Pollatsek, 1989). Fixations less than $120 \mathrm{msec}$ were eliminated from the analysis because such short fixations are thought to reflect oculomotor programming (Morrison, 1984). Fixations longer than $800 \mathrm{msec}$ were assumed to be the result of momentary track losses or eye blinks and were also eliminated. This resulted in 7\% of the data's being eliminated from the analyses. Each analysis of variance (ANOVA) was performed on the participant data $\left(F_{1}\right)$ and again on the item $\left(F_{2}\right)$ variability. 


\section{Initial Processing on the Target Word}

Gaze duration on the target word. The analyses of first-fixation duration were consistent with the analyses for gaze duration. The means for both these measures are reported in the tables, but only the analyses associated with gaze duration are reported in the text.

Topic neutral. In order to assess the impact of the target sentence context on word processing, independent of any topic information, a 2 (meaning dominance: biased vs. balanced) $\times 2$ (word type: ambiguous target vs. control word) ANOVA was performed on the topic-neutral condition. As can be seen in the topic-neutral condition in Table 2, when the target sentence context supported the subordinate interpretation of the ambiguous word, readers fixated biased ambiguous words longer than they fixated their controls (296 vs. $268 \mathrm{msec}$, respectively), and there were no significant differences between the balanced ambiguous targets and their unambiguous control words (269 vs. $275 \mathrm{msec}$, respectively). This was supported by a significant interaction in the 2 (meaning dominance: biased vs. balanced) $\times 2$ (word type: ambiguous target vs. control) ANOVA on the topic-neutral condition $\left[F_{1}(1,35)=7.28, M S_{\mathrm{e}}=1,545, p<.05\right.$, and $F_{2}(1,71)=$ $\left.6.73, M S_{\mathrm{e}}=1,847, p<.05\right]$. This replicated the subordinate bias effect (Rayner et al., 1994).

Balanced words. In order to assess the influence of topic information on word processing for a meaning dominance category (e.g., balanced), 2 (word type: ambiguous target vs. control) $\times 3$ (topic: consistent, inconsistent, and neutral) ANOVAs were conducted. For the balanced ambiguous words, no processing differences were found between the ambiguous targets and controls ( 270 vs. $275 \mathrm{msec}$, respectively) across topic conditions, since no main effect for word type (ambiguous target vs. control) was obtained $\left(F_{\mathrm{S}}<1\right)$. This effect replicated the findings from other eye movement experiments in which context preceded balanced ambiguous words (Duffy et al., 1988; Rayner \& Frazier, 1989). More important for the focus of this study, there was no main effect or interaction of topic information (all $F_{\mathrm{S}}<1$ ). Thus, there was no evi- dence that topic information influenced the initial processing of balanced ambiguous words.

Biased words. An examination of the means for the biased ambiguous words demonstrated that processing times were inflated for the ambiguous target word $(293 \mathrm{msec})$ relative to their control words $(270 \mathrm{msec})$. The 2 (word type: ambiguous target vs. control) $\times 3$ (topic: consistent, inconsistent, and neutral) ANOVA on the biased ambiguous words revealed a significant main effect of word type (ambiguous target vs. control) $\left[F_{1}(2,70)=18.49, M S_{\mathrm{e}}=\right.$ $1,575, p<.05$, and $F_{2}(2,142)=14.65, M S_{\mathrm{e}}=2,103, p<$ .05]. Importantly, topic did not affect gaze duration. There was no significant main effect for this variable, nor did topic interact with word type. Thus, the subordinate bias effect remained unchanged in the face of topic information: It was not reduced in the topic-consistent condition, in which both topic and target sentence context biased the subordinate meaning, nor was it exaggerated in the topic-inconsistent condition, in which the two sources of context conflicted.

\section{Posttarget Analyses}

The posttarget region included the area beginning immediately after the target word to the end of that sentence, and this measure was calculated by summing all consecutive fixations in that region. This region was examined to assess postaccess processing (such as meaning selection and/or integration of the meaning into the ongoing discourse representation), and the means for this measure are found in Table 3.

Biased words. In the posttarget region for the biased ambiguous words, no processing differences were found between targets and control words. The 2 (word type: ambiguous target vs. control) $\times 3$ (topic: consistent, inconsistent, and neutral) ANOVA revealed that the main effect of word type was nonsignificant $\left(F_{\mathrm{S}}<1\right)$. However, topic did affect the amount of time spent in the posttarget region $\left[F_{1}(2,70)=4.67, M S_{\mathrm{e}}=8,740, p<.05\right.$, and $\left.F_{2}(2,142)=7.32, M S_{\mathrm{e}}=6,581, p<.05\right]$. Post hoc analyses revealed that readers spent more time in the

Table 2

Mean Gaze Duration (in Milliseconds) on the Target Word, and Standard Deviations, as a Function of Discourse Topic When Target Sentence Context Biases Toward the Subordinate Interpretation (Experiment 1)

\begin{tabular}{|c|c|c|c|c|c|c|c|c|c|c|c|c|c|c|}
\hline & \multicolumn{4}{|c|}{ Topic Consistent } & \multicolumn{4}{|c|}{ Topic Inconsistent } & \multicolumn{4}{|c|}{ Topic Neutral } & \multirow[b]{3}{*}{ Gaze } & \multirow{3}{*}{$\begin{array}{c}\text { First } \\
\text { Fixation }\end{array}$} \\
\hline & \multicolumn{2}{|c|}{ Gaze } & \multicolumn{2}{|c|}{$\begin{array}{c}\text { First } \\
\text { Fixation } \\
\end{array}$} & \multicolumn{2}{|c|}{ Gaze } & \multicolumn{2}{|c|}{$\begin{array}{c}\text { First } \\
\text { Fixation } \\
\end{array}$} & \multicolumn{2}{|c|}{ Gaze } & \multicolumn{2}{|c|}{$\begin{array}{c}\text { First } \\
\text { Fixation } \\
\end{array}$} & & \\
\hline & $M$ & $S D$ & $M$ & $S D$ & $M$ & $S D$ & $M$ & $S D$ & $M$ & $S D$ & $M$ & $S D$ & & \\
\hline \multicolumn{15}{|l|}{ Biased } \\
\hline Target & 296 & 59 & 260 & 53 & 288 & 54 & 249 & 40 & 296 & 53 & 268 & 39 & 293 & 259 \\
\hline Control & $\begin{array}{l}272 \\
\mathbf{2 8 4}\end{array}$ & 44 & $\begin{array}{l}242 \\
\mathbf{2 5 1}\end{array}$ & 36 & $\begin{array}{l}271 \\
\mathbf{2 8 0}\end{array}$ & 54 & $\begin{array}{l}248 \\
249\end{array}$ & 33 & $\begin{array}{l}268 \\
\mathbf{2 8 2}\end{array}$ & 42 & $\begin{array}{l}244 \\
256\end{array}$ & 40 & 270 & 245 \\
\hline \multicolumn{15}{|l|}{ Balanced } \\
\hline Target & 272 & 57 & 249 & 38 & 269 & 42 & 248 & 30 & 269 & 52 & 239 & 29 & 270 & 245 \\
\hline Control & $\begin{array}{l}274 \\
\mathbf{2 7 3}\end{array}$ & 74 & $\begin{array}{l}240 \\
\mathbf{2 4 5}\end{array}$ & 35 & $\begin{array}{l}277 \\
\mathbf{2 7 3}\end{array}$ & 51 & $\begin{array}{l}246 \\
247\end{array}$ & 44 & $\begin{array}{l}275 \\
272\end{array}$ & 66 & $\begin{array}{l}242 \\
\mathbf{2 4 1}\end{array}$ & 50 & 275 & 243 \\
\hline
\end{tabular}

Note-Marginal means are in boldface. 
Table 3

Mean First-Pass Times (in Milliseconds) in the Posttarget Region, and Standard Deviations, as a Function of Discourse Topic When Target Sentence Context Biases Toward the Subordinate Interpretation (Experiment 1)

\begin{tabular}{|c|c|c|c|c|c|c|c|}
\hline & \multicolumn{2}{|c|}{ Topic Consistent } & \multicolumn{2}{|c|}{ Topic Inconsistent } & \multicolumn{2}{|c|}{ Topic Neutral } & \\
\hline & $M$ & $S D$ & $M$ & $S D$ & $M$ & $S D$ & \\
\hline \multicolumn{8}{|l|}{ Biased } \\
\hline Target & 610 & 126 & 695 & 85 & 644 & 70 & 650 \\
\hline Control & $\begin{array}{l}618 \\
614\end{array}$ & 125 & $\begin{array}{l}649 \\
672\end{array}$ & 73 & $\begin{array}{l}643 \\
\mathbf{6 4 4}\end{array}$ & 91 & 637 \\
\hline \multicolumn{8}{|l|}{ Balanced } \\
\hline Target & 578 & 115 & 677 & 60 & 653 & 62 & 636 \\
\hline Control & $\begin{array}{l}590 \\
\mathbf{5 8 4}\end{array}$ & 115 & $\begin{array}{l}615 \\
\mathbf{6 4 6}\end{array}$ & 60 & $\begin{array}{l}639 \\
\mathbf{6 4 6}\end{array}$ & 64 & 615 \\
\hline
\end{tabular}

Note-Marginal means are in boldface.

posttarget region in the topic-inconsistent condition relative to both topic-neutral and topic-consistent conditions $(p$ s <.05). Additionally, readers spent less time in the topic-consistent condition relative to the topic-neutral condition $(p \mathrm{~s}<.05)$. More importantly, there was a significant interaction between word type and topic $\left[F_{1}(2,70)=8.26, M S_{\mathrm{e}}=5,278, p<.05\right.$, and $F_{2}(2,142)=$ $\left.6.28, M S_{\mathrm{e}}=7,581, p<.05\right]$. Readers spent more time in this region in the topic-inconsistent condition when the ambiguous target was present relative to when the control was present in this topic condition $(p s<.05)$. However, no differences between ambiguous targets and controls were found in the other two topic conditions ( $p$ s $>$ $.05)$. Thus, when the topic information was inconsistent with the meaning of the ambiguous word biased by the sentence context, postaccess difficulty occurred.

Balanced words. In the posttarget region for the balanced ambiguous words, no processing differences were found between targets and control words. The 2 (word type: ambiguous target vs. control) $\times 3$ (topic: consistent, inconsistent, and neutral) ANOVA revealed that the main effect of word type was nonsignificant $(F \mathrm{~s}<1)$. However, topic did affect the amount of time spent in the posttarget region $\left[F_{1}(2,70)=9.39, M S_{\mathrm{e}}=5,428, p<.05\right.$, and $\left.F_{2}(2,142)=7.11, M S_{\mathrm{e}}=4,589, p<.05\right]$. Post hoc analyses revealed that readers spent more time in the posttarget region in the topic-inconsistent condition relative to the topic-consistent condition ( $p \mathrm{~s}<.05$ ). Additionally, readers spent less time in the topic-consistent condition relative to the topic-neutral condition ( $p$ s <.05), and the difference between the topic-inconsistent and topic-neutral conditions was not significant ( $p$ s $>.05$ ). More importantly, there was a significant interaction between word type and topic $\left[F_{1}(2,70)=9.15, M S_{\mathrm{e}}=4,789, p<.05\right.$, and $\left.F_{2}(2,142)=10.26, M S_{\mathrm{e}}=5,543, p<.05\right]$. Readers spent more time in this region in the topic-inconsistent condition when the ambiguous target was present relative to when the control was present in this topic condition $(p \mathrm{~s}<.05)$. However, no differences between ambiguous targets and controls were found in the other two topic conditions ( $p s>.05)$. Thus, in agreement with what was found for the biased ambiguous words, when the topic information was inconsistent with the meaning of the ambiguous word biased by the sentence context, postaccess difficulty was evident.

\section{Discussion}

The pattern of results on the initial processing measures in the topic-neutral condition replicated the findings of previous eye movement studies (Duffy et al., 1988; Rayner \& Frazier, 1989; Rayner et al., 1994). Processing times were inflated for the biased ambiguous targets relative to balanced words and the unambiguous control words, demonstrating that meaning dominance and sentence context can influence the order in which the meanings are accessed. In stark contrast, however, there was no evidence that topic information influenced the initial access stage of lexical ambiguity processing. Three pieces of evidence from Experiment 1 point to this conclusion. First, the subordinate bias effect persisted in the topic-consistent condition. Interestingly, the discourse information did not reduce initial processing difficulty even though both topic and target sentence context supported the subordinate meaning. Furthermore, the inconsistent topic information did not increase the magnitude of the subordinate bias effect relative to the topic-neutral condition. This was quite interesting, since the topic information was biased in favor of the more frequent interpretation of the ambiguous word. No changes on the initial processing measures were noted in spite of two sources of information, meaning dominance of the target word and topic information converging on the dominant interpretation. Finally, inflated initial processing times for the balanced ambiguous targets in the topic-inconsistent condition did not surface. These three pieces of evidence suggest that topic information does not influence initial stages of ambiguity processing.

Whereas early stages of processing were unaffected by topic information, later stages of ambiguity processing were influenced when the topic information was inconsistent with the bias established in the sentence containing the target word. This processing difficulty was 
Table 4

Example Passages from Experiment 2: Target Sentence Context Is Always Biased Toward Dominant Meaning

Topic Consistent

Sammy needed cash for the trip he was taking. Although he could not travel far, he was still excited. He got off work early in order to get everything together. He wrote a check at the downtown bank/shop before he left.

Topic Inconsistent

Sammy hadn't been on a fishing trip in years. Although he could not travel far, he was still excited. He got off work early in order to get everything together. He wrote a check at the downtown bank/shop before he left.

Topic Neutral

Sammy had waited a long time for this trip. Although he could not travel far, he was still excited. He got off work early in order to get everything together. He wrote a check at the downtown bank/shop before he left.

Note-The ambiguous target word is italicized in the last sentence and is followed by its matched control word.

evident in the inflated processing times in the posttarget region following both biased and balanced ambiguous words in the topic-inconsistent condition relative to their unambiguous controls. Thus, across balanced and biased ambiguous words, it appeared that postaccess difficulty occurred when the meaning of the ambiguous word, which was consistent with the target sentence context, was inconsistent with the topic information. The postaccess difficulty present in this experiment suggests that the meaning of the ambiguous word that was not biased by the target sentence context was interfering at the postaccess level. The existence of the interference of the contextually inappropriate meaning suggests that the meaning was accessed at some point. This evidence is inconsistent with a selective access account of ambiguity resolution. A selective access account suggests that the only the contextually appropriate meaning is accessed, thereby leaving the inappropriate meaning unaffected.

\section{EXPERIMENT 2}

Experiment 1 provided evidence suggesting that the subordinate meaning of an ambiguous word was not selectively accessed. However, several studies have demonstrated selective access of the dominant meaning of a biased ambiguous word (Paul et al., 1992; Tabossi, 1988; Tabossi et al., 1987; Tabossi \& Zardon, 1993). Therefore, Experiment 2 focused on the possibility of selective access of the dominant interpretation. In Experiment 2, the target sentence context favored the dominant interpretation of the ambiguous word. The topic information was consistent with the dominant meaning (topic consistent), consistent with the subordinate meaning (topic inconsistent), or neutral with respect to either meaning (topic neutral). (See Table 4 for examples.) As in Experiment 1, participants' eye movements were monitored as they read passages that contained either a balanced or a biased ambiguous word.
No processing differences were expected on the initial processing measures in Experiment 2. The target sentence context should increase the activation of the dominant interpretation in such a way that it would be accessed prior to the subordinate meaning. The occurrence of postaccess difficulty in the topic-inconsistentcondition of this experiment would provide evidence that the dominant meaning was not selectively accessed. That is, the interference would suggest that the subordinate meaning of the ambiguous word was accessed because it interfered at a later point. If the subordinate meaning of the ambiguous word was unaffected, then no interference would be expected.

\section{Method}

Participants. Thirty-six University of South Carolina students received either class credit or payment for participation in the experiment. The sample reflected a general cross section of the university community, consisting of both undergraduate and graduate students. All participants had uncorrected vision and were native English speakers.

Apparatus and Procedure. The apparatus and procedure were identical to those of Experiment 1.

Materials. The ambiguous words from Experiment 1 were used in Experiment 2. In this experiment, however, the target sentence context established a bias consistent with the dominant interpretation of the ambiguous word, and as in Experiment 1, the contextual information preceded the target word. As in Experiment 1, control conditions were created by replacing the ambiguous target words with an unambiguous word matched on length and frequency that also fit into the sentence context. The average word frequency count for the biased words was 59 per million (range: 1-242), and the average length was 4.7 characters (range: $3-7$ ); the average word frequency for their matched control words was 62 (range: $3-237$ ), and the average word length was 4.7 (range: $3-7$ ). The average frequency of the balanced ambiguous words was 54 per million (range: 1-286), and the average length was 5.1 (range: 4-7); the average frequency of their matched control words was 59 (range: 1-306), and the average length was 5.1 (range: 4-7).

In order to ensure that the ambiguous targets and control words fit equally well into the target sentence contexts, two norming tasks were conducted using the same procedures that were employed in Experiment 1. There were 30 Mount Holyoke students in each of the norming tasks. For the rating task, the means for the ambiguous targets and matched controls were 6.4 and 6.3, respectively $(p>$ $.05)$. For the test of naturalness, the mean value obtained was 0.009 . Thus these norming tasks demonstrated that both words fit equally well into the target sentence contexts.

As in Experiment 1, there were three topic conditions: The topicconsistent condition established a bias consistent with the dominant interpretation, the topic-inconsistent condition established a bias toward the subordinate interpretation, and topic-neutral did not create a bias in favor of either interpretation. Again, two filler sentences intervened between the topic-setting sentence and the sentence that contained the target word.

\section{Results}

The same processing measures and regions of text that were examined in Experiment 1 were investigated in Experiment 2 .

Initial processing on the target word. Mean gaze durations are presented in Table 5. To assess the effect of the target sentence context on word processing, indepen- 
Table 5

Mean Gaze Duration (in Milliseconds) on the Target Word, and Standard Deviations, as a Function of Discourse Topic When Target Sentence Context Biases Toward the Dominant Interpretation (Experiment 2)

\begin{tabular}{|c|c|c|c|c|c|c|c|c|c|c|c|c|c|c|}
\hline & \multicolumn{4}{|c|}{ Topic Consistent } & \multicolumn{4}{|c|}{ Topic Inconsistent } & \multicolumn{4}{|c|}{ Topic Neutral } & \multirow[b]{3}{*}{ Gaze } & \multirow{3}{*}{$\begin{array}{c}\text { First } \\
\text { Fixation } \\
\end{array}$} \\
\hline & \multicolumn{2}{|c|}{ Gaze } & \multicolumn{2}{|c|}{$\begin{array}{c}\text { First } \\
\text { Fixation } \\
\end{array}$} & \multicolumn{2}{|c|}{ Gaze } & \multicolumn{2}{|c|}{$\begin{array}{c}\text { First } \\
\text { Fixation } \\
\end{array}$} & \multicolumn{2}{|c|}{ Gaze } & \multicolumn{2}{|c|}{$\begin{array}{c}\text { First } \\
\text { Fixation } \\
\end{array}$} & & \\
\hline & $M$ & $S D$ & $M$ & $S D$ & $M$ & $S D$ & $M$ & $S D$ & $M$ & $S D$ & $M$ & $S D$ & & \\
\hline \multicolumn{15}{|l|}{ Biased } \\
\hline \multirow{2}{*}{$\begin{array}{l}\text { Target } \\
\text { Control }\end{array}$} & 279 & 42 & 244 & 49 & 278 & 46 & 240 & 45 & 275 & 87 & 246 & 47 & 277 & 243 \\
\hline & $\begin{array}{l}277 \\
\mathbf{2 7 8}\end{array}$ & 50 & $\begin{array}{l}245 \\
\mathbf{2 4 5}\end{array}$ & 38 & $\begin{array}{l}284 \\
\mathbf{2 8 1}\end{array}$ & 62 & $\begin{array}{l}248 \\
\mathbf{2 4 4}\end{array}$ & 61 & $\begin{array}{l}278 \\
277\end{array}$ & 69 & $\begin{array}{l}256 \\
\mathbf{2 5 1}\end{array}$ & 57 & 280 & 250 \\
\hline \multicolumn{15}{|l|}{ Balanced } \\
\hline Target & 278 & 87 & 258 & 64 & 278 & 73 & 262 & 63 & 287 & 89 & 263 & 52 & 281 & 261 \\
\hline \multirow[t]{2}{*}{ Control } & 286 & 81 & 266 & 62 & 281 & 71 & 258 & 50 & 288 & 86 & 263 & 58 & 285 & 262 \\
\hline & 282 & & 262 & & 280 & & 260 & & 288 & & 263 & & & \\
\hline
\end{tabular}

Note-Marginal means are in boldface.

dent of any topic information, a 2 (meaning dominance: biased vs. balanced) $\times 2$ (word type: target vs. control) ANOVA was conducted using the topic-neutral condition. There were no processing time differences between ambiguous targets and controls (281 vs. $283 \mathrm{msec}$, respectively) for biased or balanced words (all $F_{\mathrm{S}}<1$ ). In order to assess the impact of topic on word processing, a 3 (topic: consistent, inconsistent, and neutral) $\times 2$ (word type: target vs. control) ANOVA was conducted on both the biased ambiguous words and the balanced words. If topic was able to exert an early influence on word processing, inflated processing times would have been expected on the ambiguous targets in the topic-inconsistent condition relative to the topic-neutral condition for both biased and balanced words. However, the ANOVAs revealed no significant effects (all $F_{\mathrm{S}}<1$ ). This finding is consistent with the results from Experiment 1 as well as previous eye movement studies (Binder \& Morris, 1995; Rayner et al., 1994).

Posttarget analyses. The pattern of data for biased words that was obtained in the posttarget region of Experiment 1 was replicated in Experiment 2 (see Table 6 for means). In the posttarget region for the biased ambigu- ous words, no processing differences were found between targets and control words. The 2 (word type: ambiguous target vs. control) $\times 3$ (topic: consistent, inconsistent, and neutral) ANOVA revealed that the main effect of word type was nonsignificant ( $p \mathrm{~s}>.05$ ). However, topic did influence the amount of time spent in the posttarget region $\left[F_{1}(2,70)=5.11, M S_{\mathrm{e}}=9,247, p<.05\right.$, and $\left.F_{2}(2,142)=6.25, M S_{\mathrm{e}}=7,254, p<.05\right]$. Post hoc analyses revealed that readers spent more time in the posttarget region in the topic-inconsistent condition than in both the topic-neutral and the topic-consistent conditions ( $p$ s < $.05)$. Additionally, readers spent less time in the topicconsistent condition than in the topic-neutral condition $(p \mathrm{~s}<.05)$. More surprisingly, there was a significant interaction between word type and topic $\left[F_{1}(2,70)=5.36\right.$, $M S_{\mathrm{e}}=7,255, p<.05$, and $F_{2}(2,142)=4.79, M S_{\mathrm{e}}=6,992$, $p<.05]$. Readers spent more time in this region in the topic-inconsistent condition when the ambiguous target was present than when the control was present in this topic condition ( $p s<.05$ ), even though the target sentence context was biased in favor of the dominant interpretation. However, no differences between ambiguous targets and controls were found in the other two topic conditions

Table 6

Mean First-Pass Times (in Milliseconds) in the Posttarget Region, and Standard Deviations, as a Function of Discourse Topic When Target Sentence Context Biases Toward the Dominant Interpretation (Experiment 2)

\begin{tabular}{|c|c|c|c|c|c|c|c|}
\hline & \multicolumn{2}{|c|}{ Topic Consistent } & \multicolumn{2}{|c|}{ Topic Inconsistent } & \multicolumn{2}{|c|}{ Topic Neutral } & \\
\hline & $M$ & $S D$ & $M$ & $S D$ & $M$ & $S D$ & \\
\hline \multicolumn{8}{|l|}{ Biased } \\
\hline Target & 568 & 79 & 642 & 97 & 598 & 84 & 603 \\
\hline \multirow[t]{2}{*}{ Control } & 569 & 89 & 601 & 83 & 584 & 93 & 585 \\
\hline & 569 & & 622 & & 591 & & \\
\hline \multicolumn{8}{|l|}{ Balanced } \\
\hline Target & 558 & 90 & 663 & 101 & 611 & 96 & 611 \\
\hline \multirow[t]{2}{*}{ Control } & 551 & 79 & 605 & 90 & 611 & 96 & 584 \\
\hline & 555 & & 634 & & 597 & & \\
\hline
\end{tabular}

Note-Marginal means are in boldface. 
( $p$ s > .05). Postaccess difficulty occurred when the topic information was inconsistent with the instantiated meaning of the ambiguous word.

In the posttarget region for the balanced ambiguous words, no processing differences were found between targets and control words. The 2 (word type: ambiguous target vs. control) $\times 3$ (topic: consistent, inconsistent, and neutral) ANOVA revealed that the main effect of word type was nonsignificant ( $p \mathrm{~s}>.05$ ). However, topic did impact the amount of time spent in the posttarget region $\left[F_{1}(2,70)=11.22, M S_{\mathrm{e}}=6,455, p<.05\right.$, and $F_{2}(2,142)=$ $\left.8.65, M S_{\mathrm{e}}=7,528, p<.05\right]$. Post hoc analyses revealed that readers spent more time in the posttarget region in the topic-inconsistent condition than in both the topicneutral and the topic-consistent conditions $(p \mathrm{~s}<.05)$. Additionally, readers spent less time in the topic-consistent condition than in the topic-neutral condition $(p s<.05)$. Finally, there was a significant interaction between word type and topic $\left[F_{1}(2,70)=8.34, M S_{\mathrm{e}}=8,744, p<.05\right.$, and $\left.F_{2}(2,142)=6.51, M S_{\mathrm{e}}=7,254, p<.05\right]$. Readers spent more time in this region in the topic-inconsistent condition when the ambiguous target was present than when the control was present in this topic condition ( $p$ s $<$ $.05)$. However, no differences between ambiguous targets and controls were found in the other two topic conditions ( $p$ s > .05). Thus, in agreement with what was found for the biased ambiguous words, when the topic information was inconsistent with the meaning of the ambiguous word biased by the sentence context, postaccess difficulty occurred.

\section{Discussion}

As in Experiment 1, postaccess difficulty occurred when the topic information and the target sentence context were inconsistent. This difficulty was found for both biased and balanced words. This finding has implications for models of ambiguity resolution, since the interference at the discourse level is inconsistent with a selective access model. A selective access account suggests that the preceding context works to activate only the contextually appropriate meaning of an ambiguous word, leaving the inappropriate meaning unaffected, and thus it should not produce any interference. The existence of interference at the postaccess level suggests that the other meaning of the ambiguous word was accessed, since it interfered when an attempt was made to select and integrate the contextually appropriate meaning. This finding with the biased words is quite surprising, given that the meaning biased by the target sentence context information was the more frequent interpretation.

\section{GENERAL DISCUSSION}

Several studies that have investigated lexically ambiguous words have examined the role of context in the resolution process, but unfortunately, the term context has been left relatively undefined. The results of the present study set clear limits as to how and when differ- ent contextual sources of information are used in the processing of lexically ambiguous words. This study allowed for an examination of two sources of contextual information: (1) target sentence context and (2) topic information. In each experiment, the target sentence contextual information was held constant, instantiating either the subordinate meaning (Experiment 1) or the dominant interpretation (Experiment 2) of biased and balanced ambiguous words. The topic information was manipulated independently of the target sentence context so that it was consistent with the subordinate meaning or the dominant meaning, or neutral with respect to either meaning. Three important results were obtained. First, and in agreement with prior results, meaning dominance and sentence context were shown to influence initial stages of word processing. When prior sentential context preceded the ambiguous word and instantiated the subordinate sense (Experiment 1), longer processing times were found for biased ambiguous words than for balanced words or unambiguous control words. Second, topic information did not affect initial processing. The pattern of data on the initial processing measures for the topic-consistent and topic-inconsistent conditions did not differ from the pattern of data found in the topicneutral condition (Experiments 1 and 2). Third, inflated processing times were found in the topic-inconsistent condition relative to the topic-neutral condition when measures thought to reflect postaccess processes were examined (Experiments 1 and 2). These results have implications for both (1) contextual influences on ambiguity processing and (2) specific models of lexical ambiguity resolution.

\section{Evaluation of Contextual Influences on Lexical Ambiguity Processing}

The context of the critical sentence influenced the initial processing of the ambiguous words. When context preceded a balanced ambiguous word and biased toward one interpretation, the contextually appropriate meaning was accessed prior to the inappropriate meaning. For biased ambiguous words, however, when the prior context instantiated the subordinate meaning, the context boosted the activation of that meaning so that it was accessed at or near the same time as the dominant interpretation, producing inflated processing time for the ambiguous word because a time-consuming selection procedure was needed. These findings replicated those of previous eye movement research (Binder \& Rayner, 1998; Duffy et al., 1988; Rayner \& Frazier, 1989; Rayner et al., 1994).

In addition, this study allowed for an examination of the effects of discourse topic on ambiguity processing. The pattern of data in the initial processing measures in the topic-neutral condition of each experiment was replicated across the other topic conditions. That is, the subordinate bias effect was not reduced in the topic-consistent condition of Experiment 1, nor was it exaggerated in the topic-inconsistentcondition of Experiment 1, and inflated processing times were not found for the ambiguous tar- 
gets in the topic-inconsistent condition of Experiment 2. Thus, across the two experiments, there was no support for a position that suggests that discourse-level information, at least in the form of topic information, influences early stages of word processing.

Topic information did not appear to influence initial processing measures, but it did influence measures thought to reflect postaccess processes. This processing difficulty was detected in the posttarget region for both biased and balanced ambiguous words in Experiments 1 and 2. Thus, across balanced and biased ambiguous words, it appears that postaccess difficulty occurred when the meaning of the ambiguous word, which was biased by the target sentence information, was inconsistent with the topic information.

The findings from two recent ambiguity studies appear to be inconsistent with this study. Kambe et al. (2001) and Vu et al. (2000) conducted studies in which the participants read short passages in which the global context biased one meaning of an ambiguous word. However, in the critical conditions of the Kambe et al. study and all conditions of $\mathrm{Vu}$ et al., local context was absent. For example, Kambe et al. embedded biased ambiguous words in passages in which the topic sentence biased either the dominant or subordinate meaning, and although the local context always biased the subordinate meaning, it either preceded or followed the ambiguous target word. Importantly, when the local context followed the target word, but the global context was consistent with the subordinate meaning, the subordinate bias effect was obtained. Their results, as well as Vu et al.'s findings, appear to suggest that global context influences initial stages of word processing. Although these data appear to be at odds with the present study, one possible explanation is that in the absence of local context the system has to rely on global context. However, when local context is present, the major influence of global context is seen in postaccess processes, such as meaning selection and/or meaning integration.

Another possibility for the early influence may be in the choice of global information. That is, global context may be viewed as an umbrella term itself, and as such there are a number of ways to implement it. This general term may be instantiated in at least two ways: a meaningbased implementation and a structural implementation. In the present study, as well as in Kambe et al. (2001) and $\mathrm{Vu}$ et al. (2000), global context was defined as a topic, which can be classified as a meaning-based implementation. A meaning-based implementation may have to incorporate semantic relationships that exist in order to sufficiently create a bias. If this is the case, the distinction between the nature of the local and global context can become muddied. Thus, it is not surprising that some studies demonstrate an early influence of context, whereas others demonstrate a delayed influence. This discrepancy may be due to the manner in which the meaning-based global context is instantiated in the various studies. Passages from different studies may vary in (1) the number and type of semantically and/or associatively related words in the global context, and (2) the length of the intervening information between the biasing global information and the target word. Clearly, more empirical work is needed for this issue to be understood fully.

One way to eliminate the meaning-based component from a global manipulation is to use a structural manipulation. One example is to shift the structure of the discourse from one topic to another. Presumably, shifting topics makes the information associated with the previous topic less available. In fact, Binder and Morris (1995) conducted such a study. Their work demonstrated integration difficulty when the meaning of a balanced ambiguous word changed across the course of a passage, but only when the topic remained the same. When a shift in topic occurred, the processing difficulty found in the posttarget region disappeared. Thus, the data from that study are consistent with the position advocated here and illustrate structural global manipulation. Another example of a global structural manipulation is focus. Birch and Rayner (1997) linguistically focused information by using cleft sentence structures (e.g., "It was the ..." or "There was this ..."). They monitored eye movements as subjects read sentences that contained focused elements. Their results suggest that these readers were sensitive to the focus information, but that sensitivity did not materialize in measures thought to reflect initial processing. Rather, the readers made more regressions to the focused information and spent more time rereading that information. Birch and Rayner concluded that focus affects postaccess processes.

The results of the present study, in conjunction with those of past research, support the notion that local and global sources of context are distinct types of information. In addition, just as local context can be defined and implemented in a number of ways (e.g., associatively or semantically related; see Moss, Ostrin, Tyler, \& MarslenWilson, 1995, for a review), so can global context be implemented in a meaning-based or structurally based manner. Although there are exceptions, global context predominantly playes a role in postaccess processes such as meaning selection, elaboration, and/or integration.

\section{Evaluation of Models of Ambiguity Resolution}

A selective access model has difficulty accounting for the increased fixation time for biased words when the subordinate sense is instantiated (the subordinate bias effect; Rayner et al., 1994). If context aids the selection of the appropriate sense, it is unclear why the selection process would take longer for biased words. Additional evidence against a selective access account is that the subordinate bias effect persisted in the topic-consistent condition (Experiment 1). Increasing contextual bias toward the subordinate meaning of the biased ambiguous word did not reduce or eliminate the subordinate bias effect. The size of the effect obtained in the neutral contexts, where only the immediately preceding sentential context biased the ambiguous word's subordinate meaning, remained essentially unchanged when the topic of the pas- 
sage biased that meaning. Increasing the contextual bias beyond that contained in the immediately preceding sentential context did not enable selective access of the contextually appropriate meaning of the ambiguous word.

The data from Experiment 2 also challenge the selective access account. In that experiment, the target sentence context was always biased toward the dominant sense of the ambiguous word. For biased ambiguous words, the subordinate meaning should have yielded less conflict since it is much less frequently encountered than the dominant interpretation. If the dominant meaning of the ambiguous word was selectively accessed and integrated into the discourse representation, we would not have expected inflated postaccess times in the topicinconsistent condition. However, postaccess difficulty in this condition did surface, again suggesting that the subordinate meaning was activated to some degree. It is difficult to see how a selective access model could account for this finding. However, proponents of the selective access account may argue that the postaccess difficulty does not necessarily mean that the inappropriate meaning of the ambiguous word was activated. Rather, the inflated time in the posttarget region could reflect the target word's not fitting into the discourse context in the topic-inconsistent condition. However, both the ambiguous target words and the unambiguous control words were chosen to fit into the context equally well. If the postaccess difficulty were a reflection of poor semantic fit with the context, inflated processing times should be observed for both ambiguous targets and controls. This pattern was not obtained. Rather, the processing difficulty was tied to the ambiguous target words.

The findings reported from the present two experiments provide evidence consistent with the reordered access account of ambiguity resolution. First, the pattern of results in the topic-neutral condition of Experiment 1 replicated previous eye movement studies supporting the reordered access model (Binder \& Morris, 1995; Dopkins et al., 1992; Duffy et al., 1988; Rayner \& Duffy, 1986; Rayner et al., 1994; Sereno, 1995; Sereno et al., 1992). Processing times were inflated for the biased ambiguous targets relative to the balanced words and unambiguous controls when the target sentence context instantiated the subordinate interpretation (Experiment 1). The existence of the subordinate bias effect in Experiment 1 also provides evidence in favor of the reordered access model, and the postaccess measures data patterns from both experiments strengthen this position. Postaccess measures were inflated when the topic information was inconsistent with the meaning that was biased by the target sentence context. This suggests that processing difficulty occurs when the processor attempts to select and integrate a meaning of the ambiguous word that has been deemed irrelevant at the discourse level. The existence of this interference suggests that the other meaning of the ambiguous word was accessed, replicating the findings of Binder and Morris (1995).

In summary, the results from the two experiments reported here can be accommodated within the reordered access model framework, which posits that both relative meaning frequency and context influence early stages of lexical ambiguity resolution. As Simpson (1994) has noted, however, context has not been adequately specified in the ambiguity literature. The present study addressed this problem by examining more systematically the types of contexts employed and how these different forms or strengths of context influence different stages of word processing. These results suggest that sentence context influences the initial access stage, whereas discourselevel topic information influences postaccess processes such as meaning selection and integration.

\section{REFERENCES}

Binder, K. S., \& Mor r is, R. K. (1995). Eye movements and lexical ambiguity resolution: Effects of prior encounter and discourse topic. Journal of Experimental Psychology: Learning, Memory, \& Cognition, 21, 1-11.

Bin der K. S., \& Rayner, K. (1998). Contextual strength does not modulate the subordinate bias effect: Evidence from eye fixations and self-paced reading. Psychonomic Bulletin \& Review, 5, 271-276.

Birch, S., \& Rayner, K. (1997). Linguistic focus affects eye movements during reading. Memory \& Cognition, 25, 653-660.

Burgess, C., Tanen haus, M. K., \& Seidenberg, M. (1989). Context and lexical access: Implications of nonword interference for lexical ambiguity resolution. Journal of Experimental Psychology: Learning, Memory, \& Cognition, 15, 620-632.

Conrad, C. (1974). Context effects in sentence comprehension: A study of the subjective lexicon. Memory \& Cognition, 2, 130-138.

Dopkin s, S., Mor ris, R. K., \& Rayner, K. (1992). Lexical ambiguity and eye fixations in reading: A test of competing models of lexical ambiguity resolution. Journal of Memory \& Language, 31, 461-476.

Duffy, S. A., Ka mbe, G., \& Rayner , K. (2001). The effect of prior disambiguating context on the comprehension of ambiguous words: Evidence from eye movements. In D. S. Gorfein (Ed.), On the consequences of meaning selection: Perspectives on resolving lexical ambiguity (pp. 27-43). Washington, DC: American Psychological Association.

Duffy, S. A., Mor ris, R. K., \& Rayner, K. (1988). Lexical ambiguity and fixation times in reading. Journal of Memory \& Language, 27, 429-446.

Fr a ncis, W., \& Ku ̈̈er a, H. (1982). Word frequency counts of modern English. Providence, RI: Brown University Press.

Gl u cksberg, S., Kr eu z, R. J., \& Rh o, S. H. (1986). Context can constrain lexical access: Implications for models of language comprehension. Journal of Experimental Psychology: Learning, Memory, \& Cognition, 12, 323-335.

Gorfein, D. S., Viviani, J. M., \& Leddo, J. (1982). Norms as a tool for the study of homography. Memory \& Cognition, 10, 503-509.

Ka mbe, G., Rayner, K., \& Duffy, S. A. (2001). Global context effects on processing lexically ambiguous words: Evidence from eye fixations. Memory \& Cognition, 29, 363-372.

Kint sch, W., \& Mross, E. F. (1985). Context effects in word identification. Journal of Memory \& Language, 24, 336-349.

Lucas, M. M. (1987). Frequency effects on the processing of ambiguous words in sentence contexts. Language \& Speech, 30, 25-46.

Mor rison, R. E. (1984). Manipulations of stimulus onset delay in reading: Evidence for parallel programming of saccades. Journal of Experimental Psychology: Human Perception \& Performance, 10, 667682.

Moss, H. E., Ost r in, R. K., Tyl er, L. K., \& Marsl en-Wil son, W. D. (1995). Accessing different types of lexical semantic information: Evidence from priming. Journal of Experimental Psychology: Learning, Memory, \& Cognition, 21, 863-883.

Neil 1, W. T. (1989). Lexical ambiguity and context: An activationsuppression model. In D. S. Gorfein (Ed.), Resolving semantic ambiguity (pp. 63-83). New York: Springer-Verlag.

Neil 1, W. T., Hil 1 iar d, D. V., \& Cooper, E. (1988). The detection of 
lexical ambiguity: Evidence for context-sensitive parallel access. Journal of Memory \& Language, 27, 279-287.

Oden, G. C., \& Spira, J. L. (1983). Influence of context on the activation and selection of ambiguous word senses. Quarterly Journal of Experimental Psychology, 35A, 51-64.

On if er, W., \& Swinney, D. A. (1981). Accessing lexical ambiguities during sentence comprehension: Effects of frequency of meaning and contextual bias. Memory \& Cognition, 9, 225-236.

Paul, S. T., Kell as, G., Mart in, M., \& Cl ar k, M. B. (1992). The influence of contextual features on the activation of ambiguous word meanings. Journal of Experimental Psychology: Learning, Memory, \& Cognition, 18, 703-717.

Perfet t i, C. A., \& Goodman, D. (1970). Semantic constraints on the decoding of ambiguous words. Journal of Experimental Psychology, 86, 420-427.

Rayner, K., Binder, K. S., \& Duffy, S. A. (1999). Contextual strength and the subordinate bias effect. Quarterly Journal of Experimental Psychology, 52A, 841-852.

Rayner, K., \& Duffy, S. A. (1986). Lexical complexity and fixation times in reading: Effects of word frequency, verb complexity, and lexical ambiguity. Memory \& Cognition, 14, 191-201.

Rayner, K., \& Frazier, L. (1989). Selection mechanisms in reading lexically ambiguous words. Journal of Experimental Psychology: Learning, Memory, \& Cognition, 15, 779-790.

Rayner, K., Pacht, J. M., \& Duffy, S. A. (1994). Effects of prior encounter and global discourse bias on the processing of lexically ambiguous words: Evidence from eye fixations. Journal of Memory \& Language, 33, 527-544.

Rayner, K., \& Poll at sek, A. (1989). The psychology of reading. Englewood Cliffs, NJ: Prentice-Hall.

Sch vanevel dt, R. W., Meyer, D. E., \& Becker, C. A. (1976). Lexical ambiguity, semantic context and visual word recognition. Journal of Experimental Psychology: Human Perception \& Performance, 2 , 243-256.

Schwanenfl ugel, P. J., \& Whit e, C. R. (1991). The influence of paragraph information on the processing of upcoming words. Reading Research Quarterly, 26, 160-177.

Seidenberg, M. S., Tanenhau s, M. K., Leiman, J. M., \& Bienkowski, M. (1982). Automatic access of the meanings of ambiguous words in context: Some limitations of knowledge based processing. Cognitive Psychology, 14, 489-537.

Ser en o, S. C. (1995). Resolution of lexical ambiguity: Evidence from an eye movement priming paradigm. Journal of Experimental Psychology: Learning, Memory, \& Cognition, 21, 582-595.

Ser en o, S. C., Pacht , J. M., \& Rayner, K. (1992). The effect of meaning frequency on processing lexically ambiguous words: Evidence from eye fixations. Psychological Science, 3, 296-300.

Sharkey, A. J., \& Shar key, N.E. (1992). Weak contextual constraints in text and word priming. Journal of Memory \& Language, 31, 543572 .

Sharkey, N. E., \& Mit chel1, D. C. (1985). Word recognition in a functional context: The use of scripts in reading. Journal of Memory \& Language, 24, 253-270.

Simpson, G. B. (1981). Meaning dominance and semantic context in the processing of lexical ambiguity. Journal of Verbal Learning \& Verbal Behavior, 20, 120-136.

Simpson, G. B. (1984). Lexical ambiguity and its role in models of word recognition. Psychological Bulletin, 96, 316-340.

Simpson, G. B. (1994). Context and the processing of ambiguous words.
In M. A. Gernsbacher (Ed.), Handbook of psycholinguistics (pp. 359-374). San Diego: Academic Press.

Simpson, G. B., \& Krueger, M. A. (1991). Selective access of homograph meanings in sentence context. Journal of Memory \& Language, 30, 627-643.

Swinn ey, D. A. (1979). Lexical access during sentence comprehension: (Re)consideration of context effects. Journal of Verbal Learning \& Verbal Behavior, 18, 645-659.

Swinney, D. A., \& Hakes, D. T. (1976). Effects of prior context upon lexical access during sentence comprehension. Journal of Verbal Learning \& Verbal Behavior, 15, 681-689.

Tabossi, P. (1988). Accessing lexical ambiguity in different types of sentential contexts. Journal of Memory \& Language, 27, 324-340.

Tabossi, P., Col ombo, L., \& Job, R. (1987). Accessing lexical ambiguity: Effects of context and dominance. Psychological Research, 49, 161-167.

Tabossi, P., \& Zar don, F. (1993). Processing ambiguous words in context. Journal of Memory \& Language, 32, 359-372.

Tanen hau s, M. K., Leiman, J. M., \& Seiden ber g, M. S. (1979). Evidence for multiple stages in the processing of ambiguous words in syntactic contexts. Journal of Verbal Learning \& Verbal Behavior, 18, 427-440.

Til 1, R. E., Mross, E. F., \& Kint sch, W. (1988). Time course of priming for associate and inference words in a discourse context. Memory \& Cognition, 16, 283-298.

Twil l ey, L. C., Dixon, P., Tayl or, D., \& Cl ark, K. (1994). University of Alberta norms of relative meaning frequency for 566 homographs. Memory \& Cognition, 22, 111-126.

Vu, H., Kel 1 as, G., Met calf, K., \& Herman, R. (2000). The influence of global discourse on lexical ambiguity resolution. Memory \& Cognition, 28, 236-252.

\section{NOTE}

1. Creating an appropriate control condition is a debatable issue. Generally, controls have been created using three different approaches: (1) match on overall word frequency, (2) use the ambiguous word itself as a control, and (3) use a modified frequency control in which the overall word frequency is multiplied by the meaning dominance value in order to create a modified frequency count. The majority of eye movement studies have used the first approach, and the processing difficulty on the target word that has been established in these studies has been questioned. At issue here is whether that difficulty is attributable to the resolution process (i.e., accessing multiple meanings and selecting among those meanings) or whether it is due to a word frequency effect whereby the ambiguous target is of lower frequency than the control word. However, there are notable exceptions in the eye movement literature that make the frequency explanation of the processing difficulty less viable. Rayner and Frazier (1989) used the ambiguous word as the control while manipulating it in relation to the biased context. Under these conditions, they still observed processing difficulty that was attributable to the resolution process. In addition, Sereno et al. (1992) used the modified frequency approach mentioned above, and they too obtained a pattern of data that was inconsistent with a frequency explanation. For an expanded discussion on this issue, see Duffy, Kambe, and Rayner (2001).

(Manuscript received September 23, 1998; revision accepted for publication March 31, 2003.) 